\title{
Large-scale and multipolar anisotropies of cosmic rays detected at the Pierre Auger Observatory with energies above $4 \mathrm{EeV}$
}

\author{
R. M. de Almeida ${ }^{a, *}$ on behalf of the Pierre Auger $^{b}$ Collaboration \\ (a complete list of authors can be found at the end of the proceedings) \\ ${ }^{a}$ Universidade Federal Fluminense,Av. dos Trabalhadores 424, Volta Redonda, Brazil \\ ${ }^{b}$ Observatorio Pierre Auger, Av. San Martín Norte 304, 5613 Malargüe, Argentina \\ E-mail: spokespersons@auger.org
}

\begin{abstract}
More than half a century after the discovery of ultra-high energy cosmic rays (UHECRs), their origin is still an open question. The study of anisotropies in the arrival directions of such particles is an essential ingredient to solve this puzzle. We update our previous analysis of large-scale anisotropies observed by the Pierre Auger Observatory using the latest data collected before the AugerPrime upgrade. We select events with zenith angles up to 80 degrees, implying a sky coverage of $85 \%$, and energies above $4 \mathrm{EeV}$, for which the surface detector of the Observatory is fully efficient. Dipolar and quadrupolar amplitudes are evaluated through a combined Fourier analysis of the event count rate in right ascension and azimuth. The analysis is performed in three energy bins with boundaries at 4, 8, 16 and $32 \mathrm{EeV}$ and two additional cumulative bins with energies above 8 and $32 \mathrm{EeV}$. The most significant signal is a dipolar modulation in right ascension for energies above $8 \mathrm{EeV}$, as previously reported, with statistical significance of $6.6 \sigma$. Additionally, we report the measurements of the angular power spectrum for the same energy bins with the same dataset.
\end{abstract}

$37^{\text {th }}$ International Cosmic Ray Conference (ICRC 2021)

July 12th - 23rd, 2021

Online - Berlin, Germany

\footnotetext{
*Presenter
} 


\section{Introduction}

Ultra-high energy cosmic rays (UHECR) are particles with energies above $1 \mathrm{EeV}^{1}$ that hit our atmosphere coming from space. Their origin remains an open question in physics and astrophysics. The study of anisotropies in the arrival directions of UHECR as a function of energy is a very important element to unveil their sources. In particular, together with the analysis of features in the energy spectrum and information about the mass composition of these high-energy particles, it can help to understand their acceleration mechanisms at their sources and how they propagate up to Earth. Since the majority of UHECR are charged particles, they are deflected along with their propagation on magnetic field regions. The poor knowledge about the magnitude of the galactic and extragalactic magnetic fields and chemical composition of the cosmic rays makes the identification of such sources a very difficult task. Large-scale anisotropies such as dipolar or quadrupolar patterns in the flux of UHECR are expected in the case of diffusive or quasi-rectilinear propagation from an anisotropic distribution of sources or diffusive propagation from the closest extragalactic source(s). Even for a pure dipole gradient at the entrance of the Galaxy, magnetic deflections are expected to give rise to higher-order multipoles, although with small amplitude [1]. Moreover, the cosmic-ray flux could be also affected by random configurations of point sources and magnetic deflections, showing the relevance of extending the search to larger multipoles. We reconstruct the dipolar and quadrupolar components through a combined Fourier analysis of the event rate in right ascension and azimuth by assuming a pure dipolar and a dipolar plus quadrupolar flux and measure the angular power spectrum of events detected in the Pierre Auger Observatory with energies above $4 \mathrm{EeV}$.

\section{The data set}

The data set used in this work is composed of events detected with the surface detector (SD) of the Pierre Auger Observatory [2] from 2004 January 1 to 2020 December 31 with zenith angles $\theta$ up to $80^{\circ}$ and energies above $4 \mathrm{EeV}$. With this selection, we explore all the directions with declination $\delta$ between $-90^{\circ} \leq \delta \leq 45^{\circ}$, covering $85 \%$ of the sky, and exploit the fact that the SD is fully efficient for vertical events (those with zenith angles $\theta \leq 60^{\circ}$ ) with an energy above $3 \mathrm{EeV}$ and for horizontal events (those with zenith angles $\theta>60^{\circ}$ ) with an energy above $4 \mathrm{EeV}$. We consider a quality cut that requires at least five of the six water-Cherenkov detectors surrounding the station with the largest signal were operational at the time the event was recorded. The total accumulated exposure is $110,000 \mathrm{~km}^{2} \mathrm{sr} \mathrm{yr}$.

For vertical events, the energy estimation is based on the shower signal at $1000 \mathrm{~m}$ from the shower core. The latter is affected by variation of the atmospheric conditions, such as changes in the air density and pressure [3]. In particular, under hot weather conditions, the lower air densities tend to increase the lateral spread of the air shower leading to an overestimation of the primary cosmic-ray energy and ultimately to spurious daily and seasonal variations of the flux of cosmic rays above a given energy threshold. Besides, large values of the pressure correspond to an increased (decreased) matter overburden, implying that the shower is in a more (less) advanced stage when it reaches the ground level. These atmospheric effects are taken into account by correcting the energy estimator of the vertical events as described in [3]. Furthermore, the geomagnetic field breaks the

${ }^{1} 1 \mathrm{EeV} \equiv 10^{18} \mathrm{eV}$ 
circular symmetry of the shower around its axis, leading to a spurious azimuthal modulation of about $\sim 0.7 \%$, which is a posteriori corrected for vertical events following [4]. For inclined showers, the electromagnetic component is attenuated because of the larger amount of atmosphere traversed. As a result, the more penetrating muonic component is dominant making the small variations of atmospheric mass overburden negligible for their propagation, Moreover, the geomagnetic effects are accounted for a priori in the shower reconstruction. The analyses reported in this work were performed in three energy bins with boundaries at 4, 8, 16 and $32 \mathrm{EeV}$ and two additional cumulative bins with energies above 8 and $32 \mathrm{EeV}$.

\section{Analyses method and results}

\subsection{Harmonic analysis}

We perform a weighted Rayleigh analysis in right-ascension and azimuth ( $x=\alpha$ or $\phi$, respectively). The harmonic amplitudes of order $k$ are given by

$$
a_{k}^{x}=\frac{2}{\mathcal{N}} \sum_{i=1}^{N} w_{i} \cos \left(k x_{i}\right), \quad b_{k}^{x}=\frac{2}{\mathcal{N}} \sum_{i=1}^{N} w_{i} \sin \left(k x_{i}\right),
$$

where the sums run over all event $i$ and the normalization factor is $\mathcal{N}=\sum_{i=1}^{N} w_{i}$. The weight factors $w$ take into account the modulations in exposure due to the growth of the array and sporadic dead times as well as the effects of the small tilt of the array, which on average is inclined $0.2^{\circ}$ toward $\phi_{0} \simeq-30^{\circ}$. They are given by

$$
w_{i}=\left[\Delta N_{\text {cell }}\left(\alpha_{i}^{0}\right) \times\left(1+0.003 \tan \theta_{i} \cos \left(\phi_{i}-\phi_{0}\right)\right)\right]^{-1},
$$

with $\Delta N_{\text {cell }}\left(\alpha_{i}^{0}\right)$ being the normalized event count rate as a function of the right ascension of the zenith of the observatory $\alpha_{i}^{0}$ at the time the $i$-th event is recorded. The amplitudes and phases of the harmonics are given by $r_{k}^{x}=\sqrt{\left(a_{k}^{x}\right)^{2}+\left(b_{k}^{x}\right)^{2}}$ and $\varphi_{k}^{x}=\operatorname{atan}\left(b_{k}^{x} / a_{k}^{x}\right) / k$. The probability that an amplitude equal to or larger than $r_{k}^{x}$ results from fluctuations from an isotropic distribution is given by $P\left(\geq r_{k}^{x}\right)=\exp \left(-\mathcal{N}\left(r_{k}^{x}\right)^{2} / 4\right)$ [5]. The combination of first harmonic analyses in right ascension and azimuth distributions allows the reconstruction of the three components of a dipole. Assuming a pure dipolar flux, the amplitude of the dipole components in the equatorial plane $d_{\perp}$ and along the rotation axis of the Earth $d_{z}$ are given by $d_{\perp} \simeq r_{1}^{\alpha} /\langle\cos \delta\rangle$ and $d_{z} \simeq b_{1}^{\phi} /\left(\cos \ell_{o b s}\langle\sin \theta\rangle\right)$, respectively, while the dipole right ascension and declination $\left(\alpha_{d}, \delta_{d}\right)$ are given by $\alpha_{d}=\varphi_{1}^{\alpha}$ and $\delta_{d}=\operatorname{atan}\left(d_{z} / d_{\perp}\right)$. In Table 1 we show the values of the 3D dipolar reconstruction for the different energy bins considered in this work, as well as the total number of events $N$ for each energy bin and the probability that a dipole equatorial component arises by chance from an isotropic distribution. The largest departure from isotropy is for the inclusive bin above $8 \mathrm{EeV}$, for which the dipole equatorial component has a probability to arise by chance from an isotropic distribution of $5.1 \times 10^{-11}$, corresponding to a statistical significance of $6.6 \sigma$. In the left panel of Fig. 1 we present the normalized count rate as a function of the right ascension in the energy bin $E>8 \mathrm{EeV}$ with the first-harmonic modulation obtained through the Rayleigh analysis shown by a black solid line $\left(\chi^{2} /\right.$ dof $=13.06$ for 10 degrees of freedom). A map of the cosmic-ray flux for this energy 


\begin{tabular}{|c|c|c|c|c|c|c|c|}
\hline$E(\mathrm{EeV})$ & $N$ & $d_{\perp}$ & $d_{z}$ & $d$ & $\alpha_{d}\left[^{\circ}\right]$ & $\delta_{d}\left[^{\circ}\right]$ & $\mathrm{P}\left(\geq r_{1}^{\alpha}\right)$ \\
\hline $4-8$ & 106,290 & $0.01_{-0.004}^{+0.006}$ & $-0.012 \pm 0.008$ & $0.016_{-0.005}^{+0.008}$ & $97 \pm 29$ & $-48_{-22}^{+23}$ & $1.4 \times 10^{-1}$ \\
\hline $8-16$ & 32,794 & $0.055_{-0.009}^{+0.011}$ & $-0.03 \pm 0.01$ & $0.063_{-0.009}^{+0.013}$ & $95 \pm 10$ & $-28_{-13}^{+12}$ & $3.1 \times 10^{-7}$ \\
\hline $16-32$ & 9,156 & $0.072_{-0.016}^{+0.021}$ & $-0.07 \pm 0.03$ & $0.10_{-0.02}^{+0.03}$ & $81 \pm 15$ & $-43_{-14}^{+14}$ & $7.5 \times 10^{-4}$ \\
\hline$\geq 8$ & 44,398 & $0.059_{-0.008}^{+0.009}$ & $-0.042 \pm 0.013$ & $0.073_{-0.009}^{+0.011}$ & $95 \pm 8$ & $-36_{-9}^{+9}$ & $5.1 \times 10^{-11}$ \\
\hline$\geq 32$ & 2,448 & $0.11_{-0.03}^{+0.04}$ & $-0.12 \pm 0.05$ & $0.16_{-0.04}^{+0.05}$ & $139 \pm 19$ & $-47_{-15}^{+16}$ & $1.0 \times 10^{-2}$ \\
\hline
\end{tabular}

Table 1: 3D dipole reconstruction. Shown are the number of events $N$, dipole components in the equatorial plane $d_{\perp}$ and along the rotation axis of the Earth $d_{z}$, the total 3D amplitude $d$, dipole direction $\left(\alpha_{d}, \delta_{d}\right)$ and the probability to get a larger amplitude of $r_{1}^{\alpha}$ from fluctuations of an isotropic distribution.
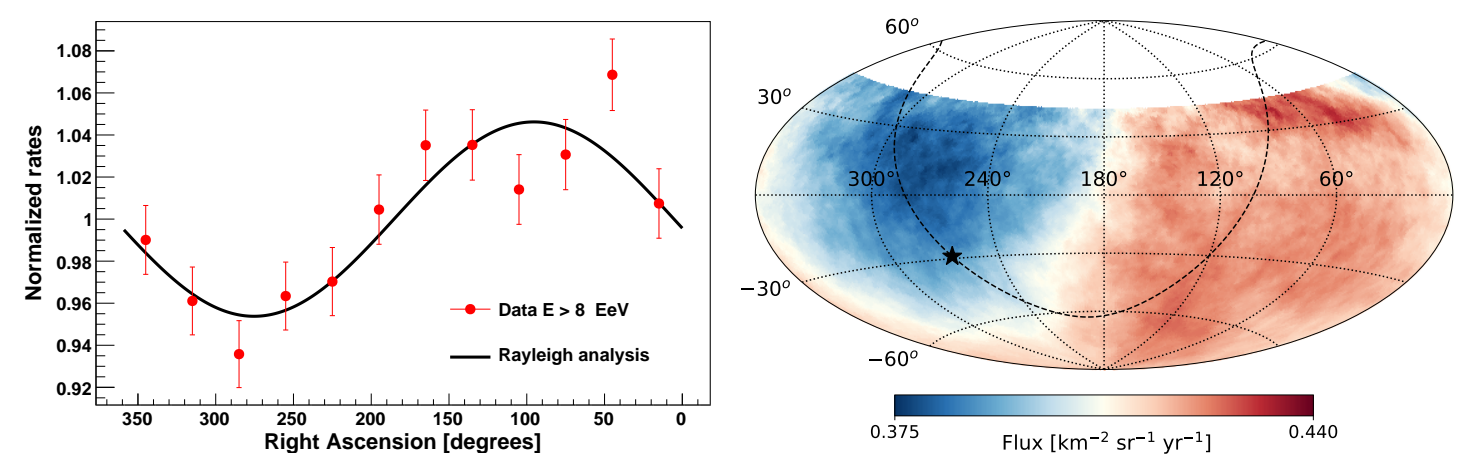

Figure 1: Left panel:. Distribution of the normalized rate of events with energy above $8 \mathrm{EeV}$ as a function of the right ascension. The first-harmonic modulation obtained through the Rayleigh analysis is shown by a black solid line. Right panel: Map of the flux of cosmic rays above $8 \mathrm{EeV}$ in equatorial coordinates averaged on top-hat windows of $45^{\circ}$ radius. The location of the Galactic plane is shown with a dashed line and the Galactic center is indicated with a star.

bin, averaged on top-hat windows of $45^{\circ}$ radius is presented in the right panel of the same figure in equatorial coordinates. The dipole direction points $\sim 115^{\circ}$ away from the direction of the Galactic centre indicating an extragalactic origin for these cosmic rays, in agreement with previous publications $[6,7]$.

The dipole amplitudes as a function of energy are presented in the left panel of Fig. 2. The evolution can be described as done in [6] by $d=d_{10}(E / 10 \mathrm{EeV})^{\beta}$ with $d_{10}=0.050 \pm 0.007$ and $\beta=0.98 \pm 0.15$. The reconstructed direction of the dipolar anisotropy for the different energy bins is shown in the right panel of Fig. 2 with corresponding $68 \%$ C.L. contours of equal probability per unit solid angle, marginalized over the dipole amplitude. There is no clear trend in the change of the dipole direction as a function of energy considering the present accuracy. The growth of the dipole amplitude as a function of energy can be a consequence of the larger relative contribution from nearby sources to the flux at higher energies with respect to the integrated flux from the more distant and isotropically distributed sources [10-18]. This suppression in the flux of sources at larges distances is expected to result from the interaction of UHECRs with the background radiation $[19,20]$. Interpretation of the reconstructed dipole directions for the different energy bins requires taking into account the magnetic deflections of the particles during their trajectory 

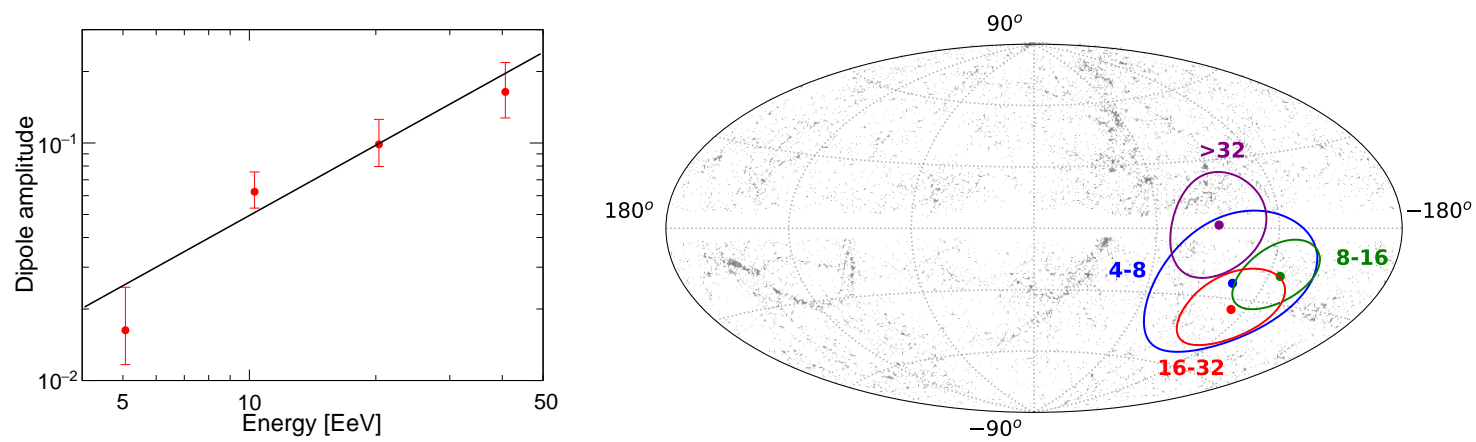

Figure 2: Left panel: Energy dependence of the dipolar amplitude measured above $4 \mathrm{EeV}$. Right panel: Reconstructed dipole directions in different energy bins and corresponding $68 \%$ C.L. uncertainty, in Galactic coordinates. The dots indicate the positions of 2MRS galaxies within $100 \mathrm{Mpc}$.

from the sources up to Earth, being a difficult task because of our still uncertain knowledge about cosmic ray composition and Galactic and extragalactic magnetic fields. Nevertheless, by using a detailed large scale structure matter density field [21] derived from the CosmicFlows-2 catalog of peculiar velocities [22], an estimation of the magnitude, direction and energy dependence of the dipolar anisotropy as a function of energy was obtained by performing a combined fit of the dipole components and cosmic ray composition [23].

Allowing for the presence of a quadrupole, the reconstructed dipolar and quadrupolar components of the flux for all energy bins were obtained as in [9] and reported in Table2. The five independent quadrupolar components are not significant in any of the energy bins.

\subsection{Angular Power Spectrum}

The angular distribution $\Phi(\mathbf{n})$ of cosmic rays observed by an experiment in some direction $\mathbf{n}$ can be decomposed by separating the dominant monopole contribution from the anisotropic one, $\Delta(\mathbf{n})$, as

$$
\Phi(\mathbf{n})=\frac{N}{4 \pi f_{1}} W(\mathbf{n})[1+\Delta(\mathbf{n})],
$$

where $W(\mathbf{n})$ is the relative coverage of the observatory, $f_{1}=\int d \mathbf{n} W(\mathbf{n}) / 4 \pi$ the fraction of the sky effectively covered by the observatory and $N$ the total number of observed cosmic rays. Unfortunately, due to the partial sky coverage of the observatory, the estimation of the individual $a_{\ell m}$ coefficients of the spherical harmonic expansion of $\Delta(\mathbf{n})$, and its angular power spectrum $C_{\ell}=\sum_{m=-\ell}^{\ell}\left|a_{\ell m}\right|^{2} /(2 \ell+1)$, cannot be carried out with relevant resolution as soon as $\ell_{\max }>$ 2. However, one can make additional assumptions ${ }^{2}$ about the ensemble-averaged expectation values of the multipole components [24] and it is possible to recover the angular power spectrum coefficients. In this situation, the pseudo-power spectrum $\tilde{C}_{\ell}=\sum_{m=-\ell}^{\ell}\left|\tilde{a}_{\ell m}\right|^{2} /(2 \ell+1)$ (which is directly measurable, obtained from $\left.\tilde{a}_{\ell m}=\int d \mathbf{n} W(\mathbf{n}) \Delta(\mathbf{n}) Y_{\ell m}(\mathbf{n})\right)$ is related to the real power spectrum through

$$
\tilde{C}_{\ell}=\sum_{\ell^{\prime}} M_{\ell \ell^{\prime}} C_{\ell^{\prime}}
$$

\footnotetext{
${ }^{2}$ For a more detailed discussion about these assumptions see [25].
} 


\begin{tabular}{|c|c|c|}
\hline Energy [EeV] & $d_{i}$ & $Q_{i j}$ \\
\hline $4-8$ & $\begin{array}{l}d_{x}=-0.008 \pm 0.007 \\
d_{y}=0.008 \pm 0.007 \\
d_{z}=-0.008 \pm 0.021\end{array}$ & $\begin{array}{l}Q_{z z}=0.008 \pm 0.036 \\
Q_{x x}-Q_{y y}=0.004 \pm 0.026 \\
Q_{x y}=-0.01 \pm 0.01 \\
Q_{x z}=-0.02 \pm 0.02 \\
Q_{y z}=-0.008 \pm 0.017\end{array}$ \\
\hline $8-16$ & $\begin{array}{l}d_{x}=-0.005 \pm 0.013 \\
d_{y}=0.045 \pm 0.013 \\
d_{z}=0.01 \pm 0.04\end{array}$ & $\begin{array}{l}Q_{z z}=0.074 \pm 0.064 \\
Q_{x x}-Q_{y y}=0.02 \pm 0.05 \\
Q_{x y}=0.039 \pm 0.024 \\
Q_{x z}=-0.002 \pm 0.031 \\
Q_{y z}=-0.03 \pm 0.03\end{array}$ \\
\hline $16-32$ & $\begin{aligned} d_{x} & =0.05 \pm 0.02 \\
d_{y} & =0.09 \pm 0.02 \\
d_{z} & =-0.15 \pm 0.07\end{aligned}$ & $\begin{array}{l}Q_{z z}=-0.14 \pm 0.14 \\
Q_{x x}-Q_{y y}=0.17 \pm 0.09 \\
Q_{x y}=-0.05 \pm 0.04 \\
Q_{x z}=0.12 \pm 0.06 \\
Q_{y z}=0.06 \pm 0.06\end{array}$ \\
\hline$\geq 32$ & $\begin{aligned} d_{x} & =-0.12 \pm 0.05 \\
d_{y} & =0.11 \pm 0.05 \\
d_{z} & =-0.22 \pm 0.13\end{aligned}$ & $\begin{array}{l}Q_{z z}=-0.17 \pm 0.26 \\
Q_{x x}-Q_{y y}=0.43 \pm 0.17 \\
Q_{x y}=0.10 \pm 0.09 \\
Q_{x z}=-0.12 \pm 0.11 \\
Q_{y z}=0.13 \pm 0.11\end{array}$ \\
\hline$\geq 8$ & $\begin{array}{l}d_{x}=-0.001 \pm 0.011 \\
d_{y}=0.06 \pm 0.01 \\
d_{z}=-0.03 \pm 0.03\end{array}$ & $\begin{array}{l}Q_{z z}=0.02 \pm 0.06 \\
Q_{x x}-Q_{y y}=0.08 \pm 0.04 \\
Q_{x y}=0.02 \pm 0.02 \\
Q_{x z}=0.02 \pm 0.03 \\
Q_{y z}=-0.003 \pm 0.026\end{array}$ \\
\hline
\end{tabular}

Table 2: Reconstructed dipole and quadrupole components for different energy bins. The $x$ axis is in the direction $\alpha=0^{\circ}$.

The operator $M$ describing the cross-talk induced by the non-uniform exposure between genuine modes is entirely determined by the knowledge of the exposure function and it is given in terms of the Wigner symbols by

$$
M_{\ell \ell^{\prime}}=\frac{2 \ell^{\prime}+1}{4 \pi} \sum_{\ell_{3}}\left(2 \ell_{3}+1\right) W_{\ell_{3}}\left(\begin{array}{ccc}
\ell & \ell^{\prime} & \ell_{3} \\
0 & 0 & 0
\end{array}\right)^{2},
$$

with the angular power spectrum of $W(\mathbf{n})$ given by $W_{\ell}=\frac{1}{2 \ell+1} \sum_{m}\left|a_{\ell m}\right|^{2}$. The power spectrum can thus be recovered from the inversion of Eq. 4. The measured power spectra for different energy bins after subtraction of the irreducible noise induced by Poisson fluctuations $\frac{4 \pi}{N} \frac{f_{1}^{2}}{f_{2}}$, with $\left.f_{2}=\int d \mathbf{n} W^{2}(\mathbf{n}) / 4 \pi\right)$, are presented in Fig.3. Besides the significant dipolar pattern corresponding to $C_{1}$, in agreement with the Rayleigh analysis, the only $C_{\ell}$ 's that stand above the $99 \%$ C.L. of isotropic fluctuations are $C_{17}$, corresponding to an angular scale of $\sim 180^{\circ} / \ell \approx 11^{\circ}$, and $C_{8}$, 

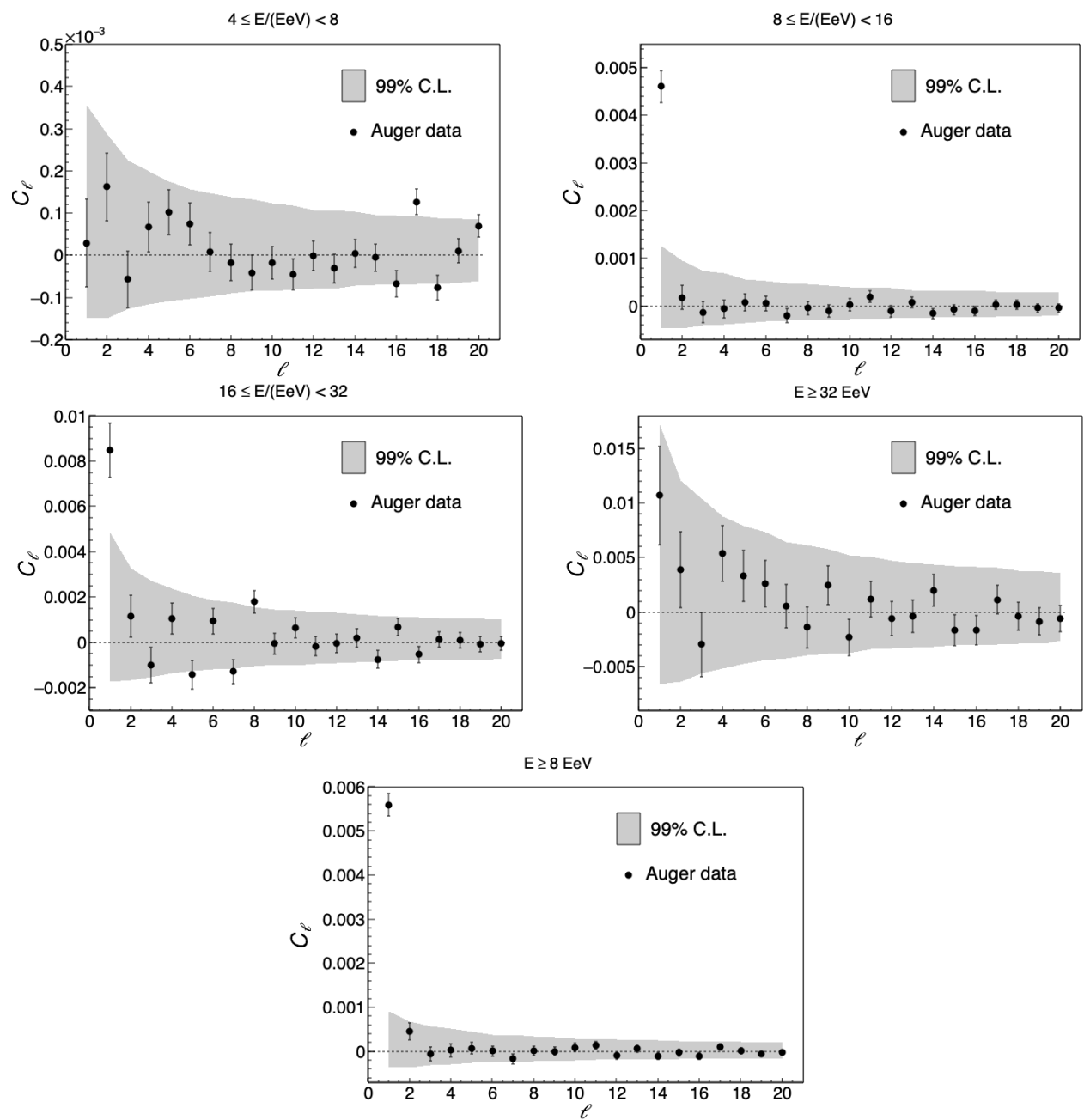

Figure 3: Angular power spectrum measurements for different energy bins. The gray bands correspond to the $99 \%$ C.L. fluctuations that would result from an isotropic distribution.

corresponding to an angular scale of $\sim 23^{\circ}$, for the energy bins $[4,8] \mathrm{EeV}$ and $[16,32] \mathrm{EeV}$, respectively. After statistical penalization for searches in different multipoles and energy bins (four independent energy bins $\times 20$ multipoles measurements $=80$ ), the statistical significances are $3.1 \%$ and $15.5 \%$, respectively. All other multipole power $C_{\ell}$ 's in different energy bins are not significant.

\section{Conclusion}

Summarizing, we updated our previous analysis of large-scale anisotropies observed by the Pierre Auger Observatory using data collected until 2020 December 31. The statistical significance of the large-scale dipolar modulation observed above $8 \mathrm{EeV}$ has increased to $6.6 \sigma$. Besides, the amplitude of the dipole increases with energies although there is no clear trend in the change of the dipole direction as a function of energy, and the quadrupole components are not significant in any of the energy bins. The $C_{1}$ obtained from the angular power spectrum increases with energy in agreement with the results obtained by using the Rayleigh analysis. All other multipoles are not 
significant. The Pierre Auger Observatory is undergoing a major upgrade phase called AugerPrime [26]. The main goal of the AugerPrime is to enhance the determination of the cosmic-ray mass composition exploiting the $100 \%$ duty cycle of the surface detectors. The additional information will certainly help the searches for anisotropies since it will allow to restrict the analyses to less deflected light particles. Therefore, the expectations for improvements in our knowledge about the sources of the ultra-high energy cosmic rays are very promising considering the detection of events by the Pierre Auger Observatory in the next years.

\section{References}

[1] D. Harari et al., J. Cosmol. Astropart. Phys. 11 (2010) 033, [1009. 5891].

[2] A. Aab et al. [Pierre Auger Coll.], Nucl. Instrum. Methods Phys. Res., Sect. A 798 (2015) 172-213, [1502 . 01323$].$

[3] A. Aab et al. [Pierre Auger Coll.], J. Instrum. 12 (2017) P02006, [1702. 02835].

[4] P. Abreu et al. [Pierre Auger Coll.], J. Cosmol. Astropart. Phys. 11 (2011) 022, [1111.7122].

[5] J. Linsley, Phys. Rev. Lett. 34 (1975) 1530-1533.

[6] A. Aab et al. [Pierre Auger Coll.], Astrophys. J. 868 (2018) 4-16, [1808.03579].

[7] A. Aab et al. [Pierre Auger Coll.], Science 357 (2017) 1266-1270, [1709.07321].

[8] E. Roulet [for the Pierre Auger Coll.], Proc. 36th Int. Cosmic Ray Conf., Madison, United States (2019), PoS(ICRC2019)358, [1909.09073].

[9] A. Aab et al. [Pierre Auger Coll.], Astrophys. J. 802 (2015) 111-122, [1411.6953].

[10] M. Giler et al., J. Phys. G: Nucl. Phys. 6 (1980) 1561-1573.

[11] V. Berezinsky et al. Astron. Astrophys. 232 (1990) 582-588.

[12] D. Harari et al., Phys. Rev. D: Part., Fields, Gravitation, Cosmol. 89 (2014) 123001, [1312 . 1366].

[13] D. Harari et al., Phys. Rev. D: Part., Fields, Gravitation, Cosmol. 92 (2015) 063014, [1507.06585].

[14] D. Wittkowski et al., Astrophys. J., Lett. 854 (2018) L3, [1710. 05617].

[15] A. di Matteo et al., Mon. Not. R. Astron. Soc. 476 (2018) 715-723, [1706. 02534].

[16] S. Hackstein et al., Mon. Not. R. Astron. Soc. 462 (2016) 3660-3671, [1607. 08872].

[17] S. Hackstein et al., Mon. Not. R. Astron. Soc. 475 (2018) 2519-2529, [1710. 01353].

[18] N. Globus et al., Astrophys. J. Lett. 850 (2017) L25, [1709. 10110].

[19] K. Greisen et al., Phys. Rev. Lett. 16 (1966) 748-750.

[20] G. T. Zatsepin et al., JETP Lett. 4 (1966) 78-80.

[21] Y. Hoffman et al., Nature Astron. 2 (2018) 680-687, [1807.03724].

[22] R. B. Tully et al., Nature 513 (2014) 71-73, [1409.0880].

[23] C. Ding et al., Astrophys. J. Lett. 913 (2021) L13, [2101.04564].

[24] O. Deligny et al., Astropart. Phys. 0410 (2004) 008, [astro-ph/0404253].

[25] A. Aab et al. [Pierre Auger Coll.], J. Cosmol. Astropart. Phys. 06 (2017) 026, [1611.06812].

[26] A. Aab et al. [Pierre Auger Coll.], [1604.03637]. 


\section{The Pierre Auger Collaboration}

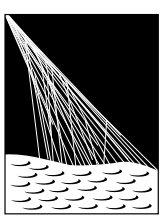

PIERRE

AUSGERVATORY

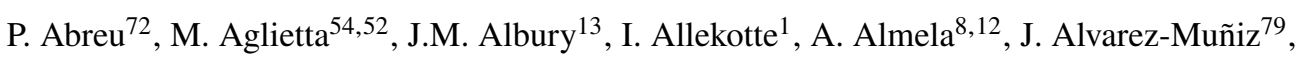
R. Alves Batista ${ }^{80}$, G.A. Anastasi ${ }^{63,52}$, L. Anchordoqui ${ }^{87}$, B. Andrada ${ }^{8}$, S. Andringa ${ }^{72}$, C. $\mathrm{Aramo}^{50}$, P.R. Araújo Ferreira ${ }^{42}$, J. C. Arteaga Velázquez ${ }^{67}$, H. Asorey $^{8}$, P. Assis ${ }^{72}$, G. Avila ${ }^{11}$, A.M. Badescu ${ }^{75}$, A. Bakalova ${ }^{32}$, A. Balaceanu ${ }^{73}$, F. Barbato ${ }^{45,46}$, R.J. Barreira Luz $^{72}$, K.H. Becker ${ }^{38}$, J.A. Bellido ${ }^{13,69}$, C. Berat ${ }^{36}$, M.E. Bertaina ${ }^{63,52}$, X. Bertou ${ }^{1}$, P.L. Biermann ${ }^{b}$, V. Binet ${ }^{6}$, K. Bismark ${ }^{39,8}$, T. Bister ${ }^{42}$, J. Biteau ${ }^{37}$, J. Blazek ${ }^{32}$, C. Bleve ${ }^{36}$, M. Boháčová ${ }^{32}$, D. Boncioli ${ }^{57,46}$, C. Bonifazi ${ }^{9,26}$, L. Bonneau Arbeletche ${ }^{21}$, N. Borodai ${ }^{70}$, A.M. Botti ${ }^{8}$, J. Brack ${ }^{d}$, T. Bretz ${ }^{42}$, P.G. Brichetto Orchera ${ }^{8}$, F.L. Briechle ${ }^{42}$, P. Buchholz ${ }^{44}$, A. Bueno ${ }^{78}$, S. Buitink ${ }^{15}$, M. Buscemi ${ }^{47}$, M. Büsken ${ }^{39,8}$, K.S. Caballero-Mora ${ }^{66}$, L. Caccianiga ${ }^{59,49}$, F. Canfora ${ }^{80,81}$, I. Caracas ${ }^{38}$, J.M. Carceller ${ }^{78}$, R. Caruso ${ }^{58,47}$, A. Castellina ${ }^{54,52}$, F. Catalanii ${ }^{19}$, G. Cataldi ${ }^{48}$, L. Cazon ${ }^{72}$, M. Cerda ${ }^{10}$, J.A. Chinellato ${ }^{22}$, J. Chudoba ${ }^{32}$, L. Chytka ${ }^{33}$, R.W. Clay ${ }^{13}$, A.C. Cobos Ceruttii ${ }^{7}$, R. Colalillo ${ }^{60,50}$, A. Coleman ${ }^{93}$, M.R. Coluccia ${ }^{48}$, R. Conceição ${ }^{72}$, A. Condorelli ${ }^{45,46}$, G. Consolati ${ }^{49,55}$, F. Contreras ${ }^{11}$, F. Convenga ${ }^{56,48}$, D. Correia dos Santos $^{28}$, C.E. Covault ${ }^{85}$, S. Dasso ${ }^{5,3}$, K. Daumiller ${ }^{41}$, B.R. Dawson ${ }^{13}$, J.A. Day ${ }^{13}$, R.M. de Almeida $^{28}$, J. de Jesús ${ }^{8,41}$, S.J. de Jong ${ }^{80,81}$, G. De Mauro ${ }^{80,81}$, J.R.T. de Mello Neto ${ }^{26,27}$, I. De Mitri ${ }^{45,46}$, J. de Oliveira ${ }^{18}$, D. de Oliveira Franco ${ }^{22}$, F. de Palma ${ }^{56,48}$, V. de Souza $^{20}$, E. De Vito ${ }^{56,48}$, M. del Río ${ }^{11}$, O. Deligny ${ }^{34}$, L. Deval ${ }^{41,8}$, A. di Matteo $^{52}$, C. Dobrigkeit ${ }^{22}$, J.C. D’Olivo ${ }^{68}$, L.M. Domingues Mendes ${ }^{72}$, R.C. dos Anjos ${ }^{25}$, D. dos Santos $^{28}$, M.T. Dova ${ }^{4}$, J. Ebr ${ }^{32}$, R. Engel ${ }^{39,41}$, I. Epicoco ${ }^{56,48}$, M. Erdmann ${ }^{42}$, C.O. Escobar ${ }^{a}$, A. Etchegoyen ${ }^{8,12}$, H. Falcke ${ }^{80,82,81}$, J. Farmer ${ }^{92}$, G. Farrar ${ }^{90}$, A.C. Fauth ${ }^{22}$, N. Fazzini ${ }^{a}$, F. Feldbusch ${ }^{40}$, F. Fenu ${ }^{54,52}$,

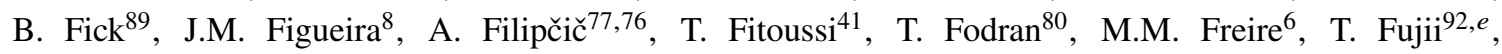
A. Fuster $^{8,12}$, C. Galea ${ }^{80}$, C. Galelli ${ }^{59,49}$, B. García ${ }^{7}$, A.L. Garcia Vegas ${ }^{42}$, H. Gemmeke ${ }^{40}$, F. Gesualdi ${ }^{8,41}$, A. Gherghel-Lascu ${ }^{73}$, P.L. Ghia ${ }^{34}$, U. Giaccari ${ }^{80}$, M. Giammarchi ${ }^{49}$, J. Glombitza ${ }^{42}$, F. Gobbi ${ }^{10}$, F. Gollan ${ }^{8}$, G. Golup ${ }^{1}$, M. Gómez Berisso ${ }^{1}$, P.F. Gómez Vitale ${ }^{11}$, J.P. Gongora ${ }^{11}$, J.M. González ${ }^{1}$, N. González ${ }^{14}$, I. Goos ${ }^{1,41}$, D. Góra ${ }^{70}$, A. Gorgi ${ }^{54,52}$, M. Gottowik ${ }^{38}$, T.D. Grubb ${ }^{13}$, F. Guarino ${ }^{60,50}$, G.P. Guedes ${ }^{23}$, E. Guido ${ }^{52,63}$, S. $\mathrm{Hahn}^{41,8}$, P. $\mathrm{Hamal}^{32}$, M.R. Hampel ${ }^{8}$, P. Hansen ${ }^{4}$, D. Harari ${ }^{1}$, V.M. Harvey ${ }^{13}$, A. Haungs ${ }^{41}$, T. Hebbeker ${ }^{42}$, D. Heck ${ }^{41}$, G.C. Hill ${ }^{13}$, C. Hojvat ${ }^{a}$, J.R. Hörandel ${ }^{80,81}$, P. Horvath ${ }^{33}$, M. Hrabovský ${ }^{33}$, T. Huege ${ }^{41,15}$, A. Insolia ${ }^{58,47}$, P.G. Isar $^{74}$, P. Janecek ${ }^{32}$, J.A. Johnsen ${ }^{86}$, J. Jurysek ${ }^{32}$, A. Kääpä ${ }^{38}$, K.H. Kampert ${ }^{38}$, N. Karastathis ${ }^{41}$, B. Keilhauer ${ }^{41}$, J. Kemp ${ }^{42}$, A. Khakurdikar ${ }^{80}$, V.V. Kizakke Covilakam $^{8,41}$, H.O. Klages ${ }^{41}$, M. Kleifges ${ }^{40}$, J. Kleinfeller ${ }^{10}$, M. Köpke ${ }^{39}$, N. Kunka ${ }^{40}$, B.L. Lago ${ }^{17}$, R.G. Lang ${ }^{20}$, N. Langner ${ }^{42}$, M.A. Leigui de Oliveira ${ }^{24}$, V. Lenok $^{41}$, A. Letessier-Selvon ${ }^{35}$, I. LhenryYvon $^{34}$, D. Lo Presti ${ }^{58,47}$, L. Lopes ${ }^{72}$, R. López ${ }^{64}$, L. Lu ${ }^{94}$, Q. Luce ${ }^{39}$, J.P. Lundquist ${ }^{76}$, A. Machado Payeras $^{22}$, G. Mancarella ${ }^{56,48}$, D. Mandat ${ }^{32}$, B.C. Manning ${ }^{13}$, J. Manshanden ${ }^{43}$, P. Mantsch ${ }^{a}$, S. Marafico ${ }^{34}$, A.G. Mariazzi ${ }^{4}$, I.C. Mariş ${ }^{14}$, G. Marsella ${ }^{61,47}$, D. Martello ${ }^{56,48}$, S. Martinelli ${ }^{41,8}$, O. Martínez Bravo ${ }^{64}$, M. Mastrodicasa ${ }^{57,46}$, H.J. Mathes ${ }^{41}$, J. Matthews ${ }^{88}$, G. Matthiae ${ }^{62,51}$, E. Mayotte ${ }^{38}$, P.O. Mazur ${ }^{a}$, G. MedinaTanco $^{68}$, D. Melo $^{8}$, A. Menshikov ${ }^{40}$, K.-D. Merenda ${ }^{86}$, S. Michal ${ }^{33}$, M.I. Micheletti ${ }^{6}$, L. Miramonti ${ }^{59,49}$, S. Mollerach ${ }^{1}$, F. Montanet ${ }^{36}$, C. Morello ${ }^{54,52}$, M. Mostafá ${ }^{91}$, A.L. Müller ${ }^{8}$, M.A. Muller ${ }^{22}$, K. Mulrey ${ }^{15}$, R. Mussa ${ }^{52}$, M. Muzio ${ }^{90}$, W.M. Namasaka ${ }^{38}$, A. Nasr-Esfahani ${ }^{38}$, L. Nellen ${ }^{68}$, M. Niculescu-Oglinzanu ${ }^{73}$, M. Niechciol ${ }^{44}$, D. Nitz ${ }^{89}$, D. Nosek ${ }^{31}$, V. Novotny ${ }^{31}$, L. Nožka ${ }^{33}$, A Nucita ${ }^{56,48}$, L.A. Núñez ${ }^{30}$, M. Palatka ${ }^{32}$, J. Pallotta ${ }^{2}$, P. Papenbreer ${ }^{38}$, G. Parente ${ }^{79}$, A. Parra ${ }^{64}$, J. Pawlowsky ${ }^{38}$, M. Pech ${ }^{32}$, F. Pedreira ${ }^{79}$, J. Pȩkala ${ }^{70}$, R. Pelayo ${ }^{65}$, J. Peña-Rodriguez ${ }^{30}$, E.E. Pereira Martins ${ }^{39,8}$, J. Perez Armand ${ }^{21}$, C. Pérez Bertolli $^{8,41}$, M. Perlin ${ }^{8,41}$, L. Perrone ${ }^{56,48}$, S. Petrera ${ }^{45,46}$, T. Pierog ${ }^{41}$, M. Pimenta ${ }^{72}$, V. Pirronello ${ }^{58,47}$, M. Platino ${ }^{8}$, B. Pont $^{80}$, M. Pothast ${ }^{81,80}$, P. Privitera ${ }^{92}$, M. Prouza ${ }^{32}$, A. Puyleart ${ }^{89}$, S. Querchfeld ${ }^{38}$, J. Rautenberg ${ }^{38}$, D. Ravignani ${ }^{8}$, M. Reininghaus ${ }^{41,8}$, J. Ridky ${ }^{32}$, F. Riehn ${ }^{72}$, M. Risse ${ }^{44}$, V. Rizi ${ }^{57,46}$, W. Rodrigues de Carvalho ${ }^{21}$, J. Rodriguez Rojo ${ }^{11}$, M.J. Roncoroni ${ }^{8}$, S. Rossoni ${ }^{43}$, M. Roth ${ }^{41}$, E. Roulet ${ }^{1}$, A.C. Rovero ${ }^{5}$, P. Ruehl ${ }^{44}$, A. Saftoiu ${ }^{73}$, F. Salamida ${ }^{57,46}$, H. Salazar ${ }^{64}$, G. Salina ${ }^{51}$, J.D. Sanabria Gomez ${ }^{30}$, F. Sánchez ${ }^{8}$, E.M. Santos ${ }^{21}$, E. Santos ${ }^{32}$, F. Sarazin ${ }^{86}$, R. Sarmento ${ }^{72}$, C. Sarmiento-Cano ${ }^{8}$, R. Sato ${ }^{11}$, 
P. Savina ${ }^{56,48,34,94}$, C.M. Schäfer ${ }^{41}$, V. Scherini ${ }^{56,48}$, H. Schieler ${ }^{41}$, M. Schimassek ${ }^{39,8}$, M. Schimp ${ }^{38}$, F. Schlüter ${ }^{41,8}$, D. Schmidt ${ }^{39}$, O. Scholten ${ }^{84,15}$, P. Schovánek ${ }^{32}$, F.G. Schröder ${ }^{93,41}$, S. Schröder ${ }^{38}$, J. Schulte ${ }^{42}$, S.J. Sciutto ${ }^{4}$, M. Scornavacche ${ }^{8,41}$, A. Segreto ${ }^{53,47}$, S. Sehgal ${ }^{38}$, R.C. Shellard ${ }^{16}$, G. Sigl ${ }^{43}$, G. Silli ${ }^{8,41}$, O. Sima ${ }^{73, f}$, R. Šmída ${ }^{92}$, P. Sommers ${ }^{91}$, J.F. Soriano ${ }^{87}$, J. Souchard ${ }^{36}$, R. Squartini ${ }^{10}$, M. Stadelmaier ${ }^{41,8}$, D. Stanca ${ }^{73}$, S. Stanič ${ }^{76}$, J. Stasielak ${ }^{70}$, P. Stassi ${ }^{36}$, A. Streich ${ }^{39,8}$, M. Suárez-Durán ${ }^{14}$, T. Sudholz ${ }^{13}$, T. Suomijärvi ${ }^{37}$, A.D. Supanitsky ${ }^{8}$, Z. Szadkowski ${ }^{71}$, A. Tapia ${ }^{29}$, C. Taricco $^{63,52}$, C. Timmermans ${ }^{81,80}$, O. Tkachenko ${ }^{41}$, P. Tobiska ${ }^{32}$, C.J. Todero Peixoto ${ }^{19}$, B. Tomé ${ }^{72}$, Z. Torrès ${ }^{36}$, A. Travaini ${ }^{10}$, P. Travnicek $^{32}$, C. Trimarelli ${ }^{57,46}$, M. Tueros ${ }^{4}$, R. Ulrich ${ }^{41}$, M. Unger ${ }^{41}$, L. Vaclavek ${ }^{33}$, M. Vacula ${ }^{33}$, J.F. Valdés Galicia ${ }^{68}$, L. Valore ${ }^{60,50}$, E. Varela ${ }^{64}$, A. Vásquez-Ramírez ${ }^{30}$, D. Veberič ${ }^{41}$, C. Ventura ${ }^{27}$, I.D. Vergara Quispe ${ }^{4}$, V. Verzi ${ }^{51}$, J. Vicha ${ }^{32}$, J. Vink $^{83}$, S. Vorobiov ${ }^{76}$, H. Wahlberg ${ }^{4}$, C. Watanabe ${ }^{26}$, A.A. Watson ${ }^{c}$, M. Weber ${ }^{40}$, A. Weindl ${ }^{41}$, L. Wiencke ${ }^{86}$, H. Wilczyński ${ }^{70}$, M. Wirtz ${ }^{42}$, D. Wittkowski ${ }^{38}$, B. Wundheiler ${ }^{8}$, A. Yushkov $^{32}$, O. Zapparrata ${ }^{14}$, E. Zas ${ }^{79}$, D. Zavrtanik ${ }^{76,77}$, M. Zavrtanik ${ }^{77,76}$, L. Zehrer ${ }^{76}$

${ }^{1}$ Centro Atómico Bariloche and Instituto Balseiro (CNEA-UNCuyo-CONICET), San Carlos de Bariloche, Argentina

${ }^{2}$ Centro de Investigaciones en Láseres y Aplicaciones, CITEDEF and CONICET, Villa Martelli, Argentina

${ }^{3}$ Departamento de Física and Departamento de Ciencias de la Atmósfera y los Océanos, FCEyN, Universidad de Buenos Aires and CONICET, Buenos Aires, Argentina

${ }^{4}$ IFLP, Universidad Nacional de La Plata and CONICET, La Plata, Argentina

${ }^{5}$ Instituto de Astronomía y Física del Espacio (IAFE, CONICET-UBA), Buenos Aires, Argentina

${ }^{6}$ Instituto de Física de Rosario (IFIR) - CONICET/U.N.R. and Facultad de Ciencias Bioquímicas y Farmacéuticas U.N.R., Rosario, Argentina

${ }^{7}$ Instituto de Tecnologías en Detección y Astropartículas (CNEA, CONICET, UNSAM), and Universidad Tecnológica Nacional - Facultad Regional Mendoza (CONICET/CNEA), Mendoza, Argentina

${ }^{8}$ Instituto de Tecnologías en Detección y Astropartículas (CNEA, CONICET, UNSAM), Buenos Aires, Argentina

${ }^{9}$ International Center of Advanced Studies and Instituto de Ciencias Físicas, ECyT-UNSAM and CONICET, Campus Miguelete - San Martín, Buenos Aires, Argentina

${ }^{10}$ Observatorio Pierre Auger, Malargüe, Argentina

${ }^{11}$ Observatorio Pierre Auger and Comisión Nacional de Energía Atómica, Malargüe, Argentina

12 Universidad Tecnológica Nacional - Facultad Regional Buenos Aires, Buenos Aires, Argentina

${ }^{13}$ University of Adelaide, Adelaide, S.A., Australia

14 Université Libre de Bruxelles (ULB), Brussels, Belgium

15 Vrije Universiteit Brussels, Brussels, Belgium

${ }^{16}$ Centro Brasileiro de Pesquisas Fisicas, Rio de Janeiro, RJ, Brazil

${ }^{17}$ Centro Federal de Educação Tecnológica Celso Suckow da Fonseca, Nova Friburgo, Brazil

${ }^{18}$ Instituto Federal de Educação, Ciência e Tecnologia do Rio de Janeiro (IFRJ), Brazil

${ }^{19}$ Universidade de São Paulo, Escola de Engenharia de Lorena, Lorena, SP, Brazil

${ }^{20}$ Universidade de São Paulo, Instituto de Física de São Carlos, São Carlos, SP, Brazil

${ }^{21}$ Universidade de São Paulo, Instituto de Física, São Paulo, SP, Brazil

${ }^{22}$ Universidade Estadual de Campinas, IFGW, Campinas, SP, Brazil

${ }^{23}$ Universidade Estadual de Feira de Santana, Feira de Santana, Brazil

${ }^{24}$ Universidade Federal do ABC, Santo André, SP, Brazil

${ }^{25}$ Universidade Federal do Paraná, Setor Palotina, Palotina, Brazil

${ }^{26}$ Universidade Federal do Rio de Janeiro, Instituto de Física, Rio de Janeiro, RJ, Brazil

${ }^{27}$ Universidade Federal do Rio de Janeiro (UFRJ), Observatório do Valongo, Rio de Janeiro, RJ, Brazil

${ }^{28}$ Universidade Federal Fluminense, EEIMVR, Volta Redonda, RJ, Brazil

${ }^{29}$ Universidad de Medellín, Medellín, Colombia

${ }^{30}$ Universidad Industrial de Santander, Bucaramanga, Colombia

${ }^{31}$ Charles University, Faculty of Mathematics and Physics, Institute of Particle and Nuclear Physics, Prague, Czech Republic

32 Institute of Physics of the Czech Academy of Sciences, Prague, Czech Republic 
33 Palacky University, RCPTM, Olomouc, Czech Republic

34 CNRS/IN2P3, IJCLab, Université Paris-Saclay, Orsay, France

${ }^{35}$ Laboratoire de Physique Nucléaire et de Hautes Energies (LPNHE), Sorbonne Université, Université de Paris, CNRSIN2P3, Paris, France

${ }^{36}$ Univ. Grenoble Alpes, CNRS, Grenoble Institute of Engineering Univ. Grenoble Alpes, LPSC-IN2P3, 38000 Grenoble, France

${ }^{37}$ Université Paris-Saclay, CNRS/IN2P3, IJCLab, Orsay, France

38 Bergische Universität Wuppertal, Department of Physics, Wuppertal, Germany

${ }^{39}$ Karlsruhe Institute of Technology (KIT), Institute for Experimental Particle Physics, Karlsruhe, Germany

${ }^{40}$ Karlsruhe Institute of Technology (KIT), Institut für Prozessdatenverarbeitung und Elektronik, Karlsruhe, Germany

${ }^{41}$ Karlsruhe Institute of Technology (KIT), Institute for Astroparticle Physics, Karlsruhe, Germany

${ }^{42}$ RWTH Aachen University, III. Physikalisches Institut A, Aachen, Germany

43 Universität Hamburg, II. Institut für Theoretische Physik, Hamburg, Germany

${ }^{44}$ Universität Siegen, Department Physik - Experimentelle Teilchenphysik, Siegen, Germany

${ }^{45}$ Gran Sasso Science Institute, L'Aquila, Italy

46 INFN Laboratori Nazionali del Gran Sasso, Assergi (L'Aquila), Italy

${ }^{47}$ INFN, Sezione di Catania, Catania, Italy

${ }^{48}$ INFN, Sezione di Lecce, Lecce, Italy

${ }^{49}$ INFN, Sezione di Milano, Milano, Italy

${ }^{50}$ INFN, Sezione di Napoli, Napoli, Italy

${ }^{51}$ INFN, Sezione di Roma "Tor Vergata", Roma, Italy

52 INFN, Sezione di Torino, Torino, Italy

53 Istituto di Astrofisica Spaziale e Fisica Cosmica di Palermo (INAF), Palermo, Italy

54 Osservatorio Astrofisico di Torino (INAF), Torino, Italy

55 Politecnico di Milano, Dipartimento di Scienze e Tecnologie Aerospaziali, Milano, Italy

56 Università del Salento, Dipartimento di Matematica e Fisica "E. De Giorgi”, Lecce, Italy

57 Università dell'Aquila, Dipartimento di Scienze Fisiche e Chimiche, L'Aquila, Italy

58 Università di Catania, Dipartimento di Fisica e Astronomia, Catania, Italy

${ }^{59}$ Università di Milano, Dipartimento di Fisica, Milano, Italy

${ }^{60}$ Università di Napoli "Federico II", Dipartimento di Fisica "Ettore Pancini”, Napoli, Italy

${ }^{61}$ Università di Palermo, Dipartimento di Fisica e Chimica "E. Segrè", Palermo, Italy

62 Università di Roma "Tor Vergata", Dipartimento di Fisica, Roma, Italy

${ }^{63}$ Università Torino, Dipartimento di Fisica, Torino, Italy

${ }^{64}$ Benemérita Universidad Autónoma de Puebla, Puebla, México

${ }^{65}$ Unidad Profesional Interdisciplinaria en Ingeniería y Tecnologías Avanzadas del Instituto Politécnico Nacional (UPIITA-IPN), México, D.F., México

66 Universidad Autónoma de Chiapas, Tuxtla Gutiérrez, Chiapas, México

${ }^{67}$ Universidad Michoacana de San Nicolás de Hidalgo, Morelia, Michoacán, México

${ }^{68}$ Universidad Nacional Autónoma de México, México, D.F., México

${ }^{69}$ Universidad Nacional de San Agustin de Arequipa, Facultad de Ciencias Naturales y Formales, Arequipa, Peru

${ }^{70}$ Institute of Nuclear Physics PAN, Krakow, Poland

${ }^{71}$ University of Łódź, Faculty of High-Energy Astrophysics, Łódź, Poland

${ }^{72}$ Laboratório de Instrumentação e Física Experimental de Partículas - LIP and Instituto Superior Técnico - IST, Universidade de Lisboa - UL, Lisboa, Portugal

73 "Horia Hulubei” National Institute for Physics and Nuclear Engineering, Bucharest-Magurele, Romania

${ }^{74}$ Institute of Space Science, Bucharest-Magurele, Romania

75 University Politehnica of Bucharest, Bucharest, Romania

76 Center for Astrophysics and Cosmology (CAC), University of Nova Gorica, Nova Gorica, Slovenia

${ }^{77}$ Experimental Particle Physics Department, J. Stefan Institute, Ljubljana, Slovenia

78 Universidad de Granada and C.A.F.P.E., Granada, Spain

${ }^{79}$ Instituto Galego de Física de Altas Enerxías (IGFAE), Universidade de Santiago de Compostela, Santiago de Compostela, Spain 
${ }^{80}$ IMAPP, Radboud University Nijmegen, Nijmegen, The Netherlands

${ }^{81}$ Nationaal Instituut voor Kernfysica en Hoge Energie Fysica (NIKHEF), Science Park, Amsterdam, The Netherlands

82 Stichting Astronomisch Onderzoek in Nederland (ASTRON), Dwingeloo, The Netherlands

${ }^{83}$ Universiteit van Amsterdam, Faculty of Science, Amsterdam, The Netherlands

${ }^{84}$ University of Groningen, Kapteyn Astronomical Institute, Groningen, The Netherlands

85 Case Western Reserve University, Cleveland, OH, USA

86 Colorado School of Mines, Golden, CO, USA

${ }^{87}$ Department of Physics and Astronomy, Lehman College, City University of New York, Bronx, NY, USA

${ }^{88}$ Louisiana State University, Baton Rouge, LA, USA

${ }^{89}$ Michigan Technological University, Houghton, MI, USA

${ }^{90}$ New York University, New York, NY, USA

${ }^{91}$ Pennsylvania State University, University Park, PA, USA

92 University of Chicago, Enrico Fermi Institute, Chicago, IL, USA

93 University of Delaware, Department of Physics and Astronomy, Bartol Research Institute, Newark, DE, USA

94 University of Wisconsin-Madison, Department of Physics and WIPAC, Madison, WI, USA

${ }^{a}$ Fermi National Accelerator Laboratory, Fermilab, Batavia, IL, USA

${ }^{b}$ Max-Planck-Institut für Radioastronomie, Bonn, Germany

${ }^{c}$ School of Physics and Astronomy, University of Leeds, Leeds, United Kingdom

${ }^{d}$ Colorado State University, Fort Collins, CO, USA

$e^{e}$ now at Hakubi Center for Advanced Research and Graduate School of Science, Kyoto University, Kyoto, Japan

$f$ also at University of Bucharest, Physics Department, Bucharest, Romania 